\title{
El sistema de salud argentino: un análisis a partir del acceso a los medicamentos
}

\author{
The Argentine health system: an analysis based on access to medicines
}

Gustavo H. Marin (https://orcid.org/0000-0002-6380-6453) ${ }^{1}$

\footnotetext{
${ }^{1}$ Departamento de Salud Comunitaria, Universidad Nacional de La Plata. Calle 60 y 120 (1900) La Plata, Provincia de Buenos Aires, Argentina.

gmarin2009@gmail.com
}

\begin{abstract}
The objective of the work is to describe features of the health system in Argentina though the way that the population access to medicines. Argentine health system reflects all the systems of the world in just one country. The National Public Health system, the Social Security and Private sectors coexist in order to attend the needs and demand of the population. Medicines are good examples to analyze how the system works. This text provides a description of each health sub-sectors in Argentina, and performs an analysis on the differences in access to medicines that patients have. Finally, it is propose recommendations for the health system to improve equity in terms of population access to medicines.
\end{abstract}

Key words Access, Medicines, Health system, Sub-sectors, Argentina
Resumen El objetivo del presente trabajo es describir las características del sistema de salud argentino a través de la manera en que las personas acceden a bienes esenciales como son los medicamentos. Argentina amalgama todos los modelos de organización sanitaria existentes el mundo. El Sistema Nacional Público de Salud, la Seguridad Social y la Medicina Privada coexisten para atender las necesidades y demandas de la población. Para analizar el funcionamiento de este Sistema tan complejo, se tomaron como ejemplo a los medicamentos. En este texto se describen los componentes del sistema de salud y se analizan las modalidades de acceso a los medicamentos que tienen los pacientes pertenecientes a cada uno de los subsectores que conforman el sistema sanitario. Finalmente, se proponen recomendaciones destinadas a mejorar la equidad en el acceso a medicamentos de la población local.

Palabras clave Acceso, Medicamentos, Sistema de salud, Subsectores, Argentina 


\section{Introduccion}

Los sistemas de salud están forjados según la ideología, la historia y la cultura de cada país ${ }^{1-3}$. Se podrían identificar al menos 3 modelos bien diferenciados de Sistemas de Salud: los sistemas nacionales de salud, la seguridad social y el sistema privado ${ }^{3}$.

Cada país, de acuerdo a su historia y a su cultura adoptó alguno de ellos para brindar atención sanitaria a su población ${ }^{1}$.

En Argentina, la nutrida migración proveniente de diversos lugares del planeta, tuvo su expresión en un complejo sistema de salud altamente fragmentado ${ }^{4,5}$. En él conviven los 3 los sistemas al mismo tiempo, y cualquier país del mundo puede hallar su propio modelo reflejado en alguno de los subsectores de Argentina ${ }^{6}$. Por ello, resulta interesante su análisis ya que a través de él, podrán exponerse las ventajas y desventajas de cada sub-sector y de cada forma de financiación ${ }^{7}$.

Por un lado existe un Sistema Nacional de Salud (SNS) que es público y universal y se financia con impuestos y rentas generales, siendo incorporado en el presupuesto nacional y de cada una de las provincias. En un país federal como Argentina, este sistema no tiene una organización central, ni una coordinación fluida entre la Nación y las Provincias para la atender a sus beneficiarios. El SNS brinda servicios desde sus efectores propios (generalmente ubicados en cada jurisdicción), aunque también compra aquellos servicios que no posee y financia algunos medicamentos y dispositivos médicos. Los beneficiarios habituales del SNS son aquellos habitantes del país que no tienen una cobertura específica (es decir que no poseen un Seguro Social o un Servicio Privado). Es decir que estas personas cuentan con una cobertura pública exclusiva, basada en una oferta de servicios disponibles para ser utilizados por cualquier habitante del país, tenga o no su ciudadanía. A este sub-sector pertenecen aproximadamente entre $40-45 \%$ de los argentinos, siendo este número fluctuante a lo largo de los años, ya que el porcentaje creciente o decreciente es acompañado por los vaivenes económicos de empleo y desocupación que sufre en forma cíclica el país.

Por otro lado existe la Seguridad Social, que es un sub-sector compuesto esencialmente por los trabajadores y sus familias, y está sostenido por aportes obligatorios que realizan los asalariados, y por contribuciones que realiza su empleador. Es un sub-sector extremadamente complejo, ya que en Argentina, existen 311 Seguros diferentes (llamados Obra Social). Doscientos noventa y dos (292) son Obras Sociales Sindicales nacionales diferentes (cada una asociada a cada rama productiva en la que desarrolla sus labores el trabajador o sus familias, aunque tiene poder de elegir). Asimismo existe Obras Sociales Nacionales como IOSFA de las Fuerzas Armadas (Instituto Obra Social de las Fuerzas Armadas y de Seguridad), el INSSJP-PAMI (Instituto Nacional de Seguridad Social para Jubilados y Pensionados/Programa de Atención Médica Integral); 24 obras sociales provinciales (los empleados públicos de cada una de las 24 provincias tienen una Obra Social Provincial); y varias obras sociales que corresponden a las Universidades Públicas Nacionales?.

La Seguridad Social, es el sector con mayor participación del sistema de salud, con prácticamente un $50-55 \%$ de los argentinos que trabajan y aportan a este sub-sector.

Finalmente existe un aseguramiento privado (habitualmente denominadas "pre-pago") dirigido al 5 y el $8 \%$ de la población con un mayor poder adquisitivo y sostenido por aportes voluntarios de aquellos individuos que desean contratar este tipo de cobertura ${ }^{3}$. Generalmente este sub-sector "compra" servicios a efectores privados; aunque algunas compañías Pre-Pagas poseen sus propios efectores.

A modo de establecer cuales son las prestaciones, bienes y servicios primordiales que la población asegurada debería acceder, se estableció por Decreto 492/1995, una canasta básica de prestaciones conocida por sus siglas $(\mathrm{PMO})^{10}$; que obligatoriamente todas las prepagas y obras sociales deben brindar a sus afiliados. Si bien las obras sociales provinciales, IOSFA o PAMI no se encuentran alcanzadas por el decreto mencionado, y no están obligadas a cumplir con el PMO, han accedido voluntariamente a respetar el mismo, razón por la cual se puede decir que el PMO es una base común que lista los derechos de los beneficiario ${ }^{10}$. Habitualmente la Superintendencia de Servicios de Salud, que es el organismo rector que regula a las Obras Sociales sindicales, actualiza las prestaciones e insumos del PMO.

\section{Impronta de la Historia}

El sistema de salud argentino sufrió numerosas fluctuaciones a lo largo de su historia, acompañando las políticas públicas de las distintas administraciones (gobiernos más "estatistas" o más "liberales") que fortalecieron o debilitaron principalmente al sub-sector público (SNS) ${ }^{11}$. Por 
ejemplo, durante la década de 1990, se pretendió instalar sin éxito, la idea de la "cuota parte" que proponía asignar a cada argentino un monto de dinero fijo para que contrate una pre-paga similar al modelo de HMO (Organización para el Mantenimiento de la Salud) de EEUU (aspecto que finalmente no prosperó en el poder legislativo, lo cual hubiera herido de muerte al sub-sector del sistema nacional/provincial público de salud) ${ }^{12}$. Durante los períodos 1945-1955; 1963-1966; 1983-1989; 2003-2015; 2020 en adelante; predominaron gobiernos que fortalecieron al Sistema Nacional de Salud y a la Seguridad Social; sustentando el acceso de la población a la atención y los servicios de dichos sub-sectores. Es difícil hallar un parámetro que permita medir el acceso a estos sistemas en un momento dado. Sin embargo, reconociendo que algunos medicamentos esenciales son un bien social que permiten modificar la historia natural de una enfermedad, nos propusimos como objetivo, analizar el acceso a estos bienes de la población en el sistema de salud argentino, y describir mediante ejemplos puntuales sobre los tipos de cobertura que brinda cada sub-sector.

\section{Metodologia}

\section{Tipo de Estudio}

Revisión descriptiva sobre la modalidad de cobertura de medicamentos en Argentina, que incluye una etapa analítica focalizada en los ejemplos aportados.

\section{Método}

Revisión bibliográfica de la literatura sobre sistema de salud argentino, organización, cobertura, acceso y utilización de servicios, a través de las bases de datos científicas Medline (Pubmed), Lilac, Dialnet y TripDatabase. Además de revisar los artículos encontrados en la búsqueda bibliográfica, se incorporaron los registros, listados de medicamentos cubiertos, informes y publicaciones de los Seguros Provinciales. Para precios de venta al público se utilizó la base Kairos Argentina. Las palabras claves usadas en las búsquedas han sido: Sistema de Salud, Argentina, Acceso, Cobertura, Seguridad Social, Obra Social, Provincia, Medicamentos. Se utilizaron los operadores booleanos AND y NOT. La estrategia de búsqueda se realizó en las diferentes bases de datos a través de los siguientes modelos de abordaje: Estrategia 1: (“System”[Mesh]) AND "Health”[-
Mesh]) AND “Argentina”[Mesh]); Estrategia 2: "Medicines"[Mesh]) AND "Access to Health Care" [Mesh]; Estrategia 3: "Coverage" [Mesh] AND "Insurance" NOT "Material Damage"[Mesh]; Estrategia 4: Social Security.

\section{Universo y muestra}

Se incorporó como población de estudio a todos los habitantes de Argentina, y como universo de estudio a los tipos de cobertura para medicamentos que dicha población declaró tener (Pública exclusiva a cobertura inespecífica; Obra Social, Pre-pagas). De ese universo se tomó una muestra aleatoria de algunos medicamentos para patologías prevalentes como hipertensión arterial, diabetes o hipotiroidismo y como dichos medicamentos fueron cubiertos por el Sistema Pública, Obra Sociales Estatales Nacionales, Obras Sociales Sindicales, Obras Sociales Provinciales, y Prepagas

Asimismo como objeto de Estudio se tomaron medicamentos para el tratamiento de patologias prevalentes (hipertension, diabetes), y dentro de ellos aquellas marcas cuyo precio de venta al público sea de un valor intermédio entre el más barato y el más caro.

\section{Variables de Análisis}

Se tomaron como variables de análisis al tipo de cobertura (publica, seguridade social, prepaga), discriminado dentro de la variable "Seguridad Social" si el seguro es nacional Estatal, Provincial o Sindical, tipo de medicamento (para patología prevalente), precio de venta al público de dichos medicamentos, porcentaje del valor nominal cubierto por cada tipo de asegurador; y porcentaje de pago de bolsillo requerido para acceder al bien.

\section{Análisis estadístico}

El análisis estadístico se realizó con el software estadístico EPI INFO 7.0, cada variable fue codificada para la gestión del programa, con su descripción y sus categorías. Este programa permitió expresar los resultados en frecuencia y porcentaje de cada variable de estudio.

\section{Resultados}

Argentina es uno de los pocos países que aún tienen un sistema de salud universal. El Sub-Sec- 
tor Público (Sistema Nacional de Salud), brinda atención sin miramientos a quien lo solicite. Claro está que existen muchas limitaciones del sistema, que solo en algunos usuarios, son compensadas por otros tipo de cobertura (Obra social o Pre-paga), lo cual se trasunta en una inequidad en el acceso a bienes y prestaciones.

\section{Cobertura de medicamentos desde el sub-sector público de salud}

La crisis económica argentina del 2001-2002 fue devastadora para todos los sectores sociales y productivos, pero dejó algunos elementos positivos que aún hoy perduran.

La rápida respuesta del Estado ante las necesidades de salud más acuciantes de la población, llegó a través de los programas sanitarios como el Plan Nacer o el Remediar, que garantizan aún hoy derechos a la atención y al acceso a medicamentos esenciales para aquel sector más vulnerable de la comunidad ${ }^{13}$. En el caso del Remediar, programa creado en 2002 y vigente hasta nuestros días, transfiere en forma mensual 50 medicamentos esenciales para tratar el $80 \%$ de las patologías prevalentes del país, a más 7000 efectores (Centros de Atención Primaria de la Salud-CAPS) de todo el país para ser dispensados en forma gratuita, según la cantidad de beneficiarios que requieran esos medicamentos en cada uno de esos efectores ${ }^{14}$. Si bien esa cantidad de fármacos puede resultar insuficiente para las necesidades de la población; el programa Remediar contempla que otros financiadores públicos (por ejemplo las provincias o municipios) aporten una cantidad de unidades de esos mismos fármacos $\mathrm{u}$ otros que no se encuentren en ese listado provisto, para poder así complementar la oferta que permita atender la demanda en su totalidad, aspecto que no siempre ocurre.

La equidad en la atención en Argentina sanitaria a través del acceso de la población a los medicamentos, está reflejada en las vacunas del calendario nacional, ya que son de provisión gratuita, distribución homogénea y dispensada en forma universal para todos los habitantes del país que lo requieran.

Por otro lado, para enfermedades crónicas como la Diabetes, Epilepsia o SIDA; si bien existen leyes nacionales que obligan al Ministerio de Salud de la Nación o las provincias que adhieren a estas leyes, a proveer medicamentos en forma gratuita a las personas que se encuentran con estas afecciones; existen marcadas diferencias en cuanto a los principios activos que son cubiertos en cada jurisdicción, así como una gran variabilidad en la disponibilidad y continuidad de esas dispensas ${ }^{15}$.

Si tomamos como ejemplo a la Diabetes Mellitus, observamos que la Encuesta Nacional de Factores de Riesgo -ENFR ${ }^{16}$, detectó una prevalencia de un $10 \%$ del total de la población Argentina, aunque son muchos menos (3\%) los que se reconocen con esta patología y concurren a algún servicio de salud para el control de su enfermedad. El acceso a estos medicamentos de pacientes con cobertura exclusiva del sub-sector público, depende entonces de su inclusión en los programas vigentes. En Argentina, la Ley Nacional de Diabetes No 26.914 (2013) ampliada de una la ley original del año 1989, garantiza la cobertura del 100\% del tratamiento de la enfermedad por parte del sistema de salud, sea público o privado. No obstante, al ser un país federal, cada provincia adhirió a dicha ley con otra ley, la cual puede diferir tanto en la cobertura como en la modalidad y tipos de servicios que brinda ${ }^{17}$. Es decir que una persona sin cobertura específica que padezca esta enfermedad puede acceder diversos tratamientos según el lugar de Argentina donde resida. En la provincia de Buenos Aires, por ejemplo, solo entre un $25 \%{ }^{18}$ y un $26.3 \%{ }^{19}$ de los enfermos diabéticos que tienen cobertura pública exclusiva y que demandaron ser atendidos, tendrán acceso a la medicación que necesitan por medio de alguno de los efectores estatales. Un $8.4 \%$ de estos medicamentos serán provistos por Programa Nacional Remediar; un $87 \%$ a través del Programa Provincial (PRODIABA) (Ley 11.620), y un 4.6\% a través un apoyo desde el ámbito municipal ${ }^{19}$. En relación al tipo de fármaco cubierto, sabemos que el programa Remediar entrega Glibenclamida $5 \mathrm{mg}$ o Metformina 500 mg, 850 mg; el PRODIABA brinda cobertura para glibenclamida, metformina, insulina cristalina, insulina NPH, glargina, detemir, e insulina Lispo) y los programas municipales varían de medicamentos según el municipio. Es decir que la provincia de Buenos Aires tiene 135 municipios, con diferentes tipos de coberturas y medicamentos cubiertos por cada uno; y en forma global se registra un consumo medido en DHD de 0,14 para metformina; para glibenclamida 0,$31 ; y$ para otros hipoglucemiantes $0,45^{20}$.

En otras provincias como en Córdoba, basándonos en cifras de la ENFR ${ }^{16}$ y del programa provincial de diabetes PROCORDIA (LEY 8.785/99), un $11 \%$ de los ciudadanos diabéticos con cobertura pública exclusiva que viven en dicha provincia, son provistos con insulina (cristalina/NPH), 
glicenclamida/glicazida y metformina ${ }^{21}$. El consumo de medicamentos estos antidiabéticos por este plan provincial medido en DHD es para insulina NPH de 6,244; para insulina corriente 1,990; para Metformina de 2,497, para Glibenclamida de 2,826; y para Gliclazida de 0,281 ${ }^{22}$.

Todas estas desigualdades sumadas a otros elementos socioeconómicos, se trasuntan en una evolución diferencial de la morbi-mortalidad para cada habitante y cada región. La mortalidad anual por diabetes ajustada por edad en Argentina es del 2,6\%, sin embargo es 1,9\% en Ciudad Buenos Aires y $8 \%$ en algunas provincias ${ }^{23}$.

En el caso de patologías oncológicas, desde el sub-sector público existe provisión gratuita de medicamentos a partir de los Ministerios de Salud de cada una de las 24 provincias del país, aspecto que trae grandes inequidades, no sólo por las diferencias existentes en los listados de fármacos provistos por cada distrito, sino también por la forma y celeridad en la que se gestiona la solicitud y la dispensa de los mismos. La accesibilidad medida en términos de tasa uso de fármacos antineoplásicos cada 10000/habitantes/ año indica que provincias como Mendoza o Córdoba la misma es $>$ a 10 mientras que otras como Misiones la tasa de uso es de $4.3^{24}$. Y a ello debemos sumar la inequidad existente al interior de cada una de dichas provincias, ya que se observa una amplia diferencia en la tasa de uso según la localidad a la que pertenece el beneficiario ${ }^{24}$.

Es decir que en Argentina, aquellas personas que no cuentan con una cobertura específica en salud, el Estado en sus diferentes jurisdicciones da respuesta (en mayor o menor medida y con amplias inequidades), a una demanda de algunos medicamentos para tratar determinadas patologías que afectan a la mayor parte de la población como son las enfermedades crónicas, las patologías oncológicas o infecciosas.

Sin embargo, existe un vacío legal para la cobertura de ciertas patologías, que por no mencionarse en ninguna ley ni estar incluidas en ningún programa, la jurisdicción nacional o provincial no se hacen cargo de su provisión. Basa nombrar a enfermedades ligadas a la población más socialmente vulnerable, como son la sarna o la pediculosis; para reconocer una enorme heterogeneidad y una gran discrecionalidad en el tipo de cobertura que se brinda cada jurisdicción a este tipo de problemas desde el sub-sector público.

\section{Cobertura de medicamentos desde la seguridad social}

En Argentina, como se mencionó en la introducción, más del $50 \%$ de la población posee algún tipo de Seguridad Social (Obra Social), es decir que un $6 \%$ del salario y un 3\% de contribución del empleador se destinan a financiar la cobertura en salud.

Existen en el país numerosas obras sociales, algunas de las cuales son de gestión estatal nacional (PAMI), otras destinadas atender la población de Fuerzas Armadas y Seguridad (IOSFA), algunas a empleados de alguna de las Universidades Nacionales (DOSUBA, DASPU); otras para cuidar de la salud de los empleados públicos de cada una de las 24 provincias llamadas "Obras Sociales Provinciales" (aunque cada una tiene un nombre según la jurisdicción a la cual pertenezca); y existen otras (n292) que son las llamadas "Obras Sociales Sindicales" destinadas a brindar cobertura a los empleados de cada uno de los gremios que componen ese sector. Estas últimas obras sociales (sindicales) deben adherir a un Programa Médico Obligatorio $(\mathrm{PMO})^{10}$ en el cual existen una serie de patologías que deben ser obligatoriamente cubiertas por los financiadores de las Obras Sociales Sindicales, incluyendo los medicamentos para el tratamiento de las mismas, con un porcentaje mínimo del 40\% del valor del precio de venta al público (PVP), debiendo los afiliados aportar de su bolsillo el $60 \%$ para acceder a dichos fármacos.

Cabe aclarar que este PMO no se actualiza oficialmente en forma regular y, debido a ello, cada Obra Social interpreta sus obligaciones de manera distinta ${ }^{10}$. Así, cada uno de estos financiadores tiene su propia lista de medicamentos, $\mathrm{y}$ por lo tanto, no existe un acceso igualitario para más del 50\% de la población argentina a la terapéutica farmacológica.

Para dar algunos ejemplos de esta aseveración, podemos analizar el PAMI (obra social para jubilados y pensionados), que es el seguro social más grande de Sud-América con más de 5 millones de afiliados, brindando cobertura de los medicamentos en un porcentaje fijo del precio de venta al público (PVP) de los mismos (generalmente superior a lo establecido por el PMO), mientras que el monto restante lo debe aportar cada paciente, hecho que es preocupante, ya que la gran mayoría de los beneficiarios son ex trabajadores mayores de 65 años que tiene ingresos jubilatorios limitados. Es justo reconocer que en el año 2020 hubo avances importantes en este sentido por parte de la nueva administración de 
PAMI, ya que ha decidido dar cobertura del 100 $\%$ a 170 fármacos considerados esenciales, beneficio que es considerado primordial a la hora de garantizar los derechos de los afiliados a acceder a tratamientos para enfermedades que pueden resultar invalidantes o mortales ${ }^{25}$.

IOSFA, la Obra Social Nacional para el personal de las Fuerzas Armadas y de Seguridad con casi 600 mil afiliados, no está alcanzada por el Decreto del PMO, sin embargo adhiere al mismo, por lo tanto respeta el $40 \%$ de cobertura mínima para un amplio listado de medicamentos incluido en su listado, aunque para afiliados que consuman en farmacias propias de la Obra Social, el descuento es del $50 \%$.

IOMA (Instituto Obra Médico Asistencial) es la obra social provincial más grande, con 2.640.000 afiliados, y al igual que las otras obras sociales provinciales, cubre parcialmente la mayoría de los medicamentos de sus afiliados, pero al no tener una obligación de seguir las pautas del PMO, el porcentaje del precio de venta al público (PVP) que reconoce de alguno de ellos es variable y a veces mucho menor al $40 \%$. Esto ocurre porque la cobertura es por un monto fijo del valor de medicamento y no un porcentaje. Por lo tanto, inicialmente el valor que reconoce cubrir del precio total del medicamento es adecuado y justo, pero al aumentar el PVP, (aspecto que es frecuente en un país con elevada inflación como es Argentina), el porcentaje que reconoce la Obra Social a sus beneficiarios cae a valores sub-óptimos, a excepción de algunos planes con mayor cobertura como los antidiabéticos, con los cuales se alcanza un consumo en general medidos en DHD, de 12.76 metformina, gliclazida 0.06 , glimepirida 3.75 , glibenclamida 1.31 , insulina cristalina 0.058 , insulina lispro 0.12 , insulina aspártica 0.39 , insulina glulisina 0.044 , insulina NPH 0.50 , insulina glargina 0.26 , insulina detemir $0.093^{26}$.

Si se analizan otros ejemplos concretos de cobertura por parte de la Seguridad Social, podemos observar que para un medicamento antihipertensivo clásico como enalapril (considerando un tratamiento estándar según DDD WHO-ATC de $10 \mathrm{mg} /$ día por 30 días), hidroclorotiazida ( 25 $\mathrm{mg} /$ dia por 30 días) o losartan (50 mg/día por 30 días), para productos considerados de referencia para la agencia reguladora nacional y considerando un valor intermedio de mercado (Enalapril marca G, Hidroclorotiazida marca B, o Losartan marca " $R$ "); veremos la disparidad de cobertura que existe entre los distintos sub-sectores de la Seguridad Social. (Tabla 1)
Por otro lado, la Seguridad Social (y todos los sub-sectores y Obras Sociales que la componen) no siguen la misma lógica y suelen incorporar medicamentos en sus listados que no siempre tiene probada eficacia, que no son los más seguros, o bien no son los menos onerosos. Muestra de esta observación es que por ejemplo PAMI, IOMA, IOSFA y varias obras sociales sindicales sigue hoy cubriendo clonixinato de lisina (medicamento de dudosa eficacia) o cinarizina comprimidos de $100 \mathrm{mg}$ (medicamento inseguro a dicha dosis en población de adultos mayores) o bien anticonceptivos de mayor riesgo tromboembólico como drospirenona, desogestrel o gestodeno teniendo varias opciones mucho más seguras como levonorgestrel en el mercado farmacéutico ${ }^{27}$. Derivar un gasto innecesario en la cobertura de estos medicamentos redunda en no poder aumentar la cobertura para aquellos fármacos que tienen un impacto altamente positivo en la salud de los afiliados.

Otro elemento que hemos observado en la Seguridad Social, es que el porcentaje de cobertura resulta igual en aquellos fármacos de mayor y menor precio; sin orientar a prescriptores o usuarios a utilizar aquellos de menor costo, como lo indica la política de $\mathrm{URM}^{7}$. Por ejemplo, en medicamentos utilizados en tratamientos anti-hipertensivos, el porcentaje de cobertura es igual para el losartán que para los "me too" (fármacos con similar estructura química, idéntica eficacia y seguridad, pero habitualmente con mayor precio que el medicamentos de referencia). Dentro del mismo grupo terapéutico de bloqueantes de receptores ATII, existen productos con un valor de mercado hasta 6 veces mayor que el medicamento de referencia losartán; siendo el precio de mercado de losartán de $\$ 953$ (10.5 USD) y el de telmisartán es de $\$ 5873$ (65.3 USD). En ambas situaciones la seguridad social cubre un 40 o $50 \%$ de 65 USD y de 10 USD. Si se cubriera el 100\% de la opción más barata, no solo resultaría un beneficio para los pacientes sino también para el financiador, ya que aun pagando por el $100 \%$ del producto ahorraría 22 USD por tratamiento y por cada afiliado ${ }^{27}$. Este ejemplo brindado para los antagonistas de los receptores de angiotensina II se observa en todos los grupos terapéuticos.

En el caso de los financiadores que no cubren un porcentaje del precio de los medicamentos sino un monto fijo, como es el caso de la Obra Social Provincial IOMA, no solo existe la misma irracionalidad que las otras Obras Sociales sobre brindar cobertura a medicamentos sin probada eficacia o seguridad, sino que el impacto de la 
Tabla 1. Cobertura de cada financiador de la Seguridad Social sobre el precio total de venta al publico.

\begin{tabular}{|c|c|c|c|c|c|c|c|c|c|c|c|}
\hline \multirow[b]{2}{*}{ Producto } & \multirow{2}{*}{$\begin{array}{l}\text { PRECIO } \\
\text { PVP }\end{array}$} & \multicolumn{2}{|c|}{ PAMI $^{*}$} & \multicolumn{2}{|c|}{$\begin{array}{c}\text { Obra Social } \\
\text { Sindical }\end{array}$} & \multicolumn{2}{|c|}{$\begin{array}{c}\text { Obra Social } \\
\text { Provincial IOMA }\end{array}$} & \multicolumn{2}{|c|}{$\begin{array}{c}\text { Obra Social } \\
\text { Provincial (LR) }\end{array}$} & \multicolumn{2}{|c|}{$\begin{array}{c}\text { Obra Social } \\
\text { Provincial (SGE) }\end{array}$} \\
\hline & & $\begin{array}{c}\text { \$ a } \\
\text { cargo } \\
\text { de OS }\end{array}$ & $\begin{array}{c}\$ \text { a } \\
\text { cargo } \\
\text { paciente }\end{array}$ & $\begin{array}{c}\$ \text { a } \\
\text { cargo } \\
\text { de OS }\end{array}$ & $\begin{array}{c}\text { \$a } \\
\text { cargo } \\
\text { paciente }\end{array}$ & $\begin{array}{c}\$ \text { a } \\
\text { cargo } \\
\text { de OS }\end{array}$ & $\begin{array}{c}\$ \text { a } \\
\text { cargo } \\
\text { paciente }\end{array}$ & $\begin{array}{c}\text { \$ a cargo } \\
\text { de OS }\end{array}$ & $\begin{array}{c}\$ \text { a } \\
\text { cargo } \\
\text { paciente }\end{array}$ & $\begin{array}{c}\text { \$a } \\
\text { cargo } \\
\text { de OS }\end{array}$ & $\begin{array}{l}\$ \text { cargo } \\
\text { paciente }\end{array}$ \\
\hline Enalapril "G" & 544.98 & 481.86 & 63.12 & 217.99 & 326.99 & 197.40 & 347.58 & $\begin{array}{r}\text { Sin } \\
\text { cobertura } \\
\star \star\end{array}$ & 544.98 & 272.49 & 272.49 \\
\hline $\begin{array}{l}\text { Hidroclorotiazida } \\
\text { "B" }\end{array}$ & 866.34 & 633.06 & 233.28 & 346.53 & 519.81 & 296.10 & 570.24 & 346.53 & 519.81 & 433.17 & 433.17 \\
\hline Losartan "R" & 1260.64 & 1076.03 & 184.61 & 504 & 756.64 & 502.96 & 757.68 & $\begin{array}{r}\text { Sin } \\
\text { cobertura } \\
\star \star \star\end{array}$ & 1260.64 & 441.224 & 819.41 \\
\hline
\end{tabular}

PVP: Precio de venta al público. ${ }^{*}$ PAMI: Obra Social de Pensionados y Jubilados (cabe reconocer que estos medicamentos fueron incluidos n 2020 en listado de medicamentos esenciales cubiertos al $100 \%) .{ }^{*}$ Sin cobertura para estos productos, aunque cubre otras marcas de ese mismo principio activo al $40 \%$ del valor del PVP.

Fuente: Elaboracion propia.

inflación y la poca e incierta regularidad con la cual se actualizan los montos fijos de cobertura, provocan que los beneficiarios tengan que poner cada vez más dinero de sus bolsillos para poder acceder a ese bien esencial. Un ejemplo de ello es que en la actualidad, la cobertura sobre el valor de PVP de levo-tiroxina es del 31\%, mientras que para ciertos antihipertensivos un 22 al 50\% ${ }^{28}$. Esto resulta irracional ya que este mismo financiador tiene una cobertura del $100 \%$ para las consecuencias estas últimas enfermedades (Accidentes Cerebro-vasculares, Infartos), las cuales se ven potenciadas por el abandono de los tratamientos.

\section{Cobertura privada pre-pagas}

Las prepagas también deben obligatoriamente cubrir el mínimo del 40\% del PVP del listado de medicamentos que establece el PMO y en caso de enfermedades crónicas como diabetes o HTA el $70 \%{ }^{10}$. No obstante, existe una diferencia de cobertura según el plan elegido por el beneficiario, con distintos porcentajes a cargo del paciente según la capacidad de pago de quien contrata el servicio.

Como en el sub-sector de la Seguridad Social, aquí también existe la exigencia de un pago de bolsillo por parte de los afiliados, lo cual podría ser una barrera para el acceso, que colabore en discontinuar sus tratamientos, quedando expuestos a las consecuencias ominosas de las enfermedades que padecen.

\section{Discusion}

La Organización Mundial de la Salud, en la Conferencia de Expertos en Nairobi ${ }^{20}$, puso en primer plano de la agenda internacional, la utilización de los medicamentos, acuñando el concepto de Uso Racional de Medicamentos (URM) que significa que los pacientes deben recibir la medicación adecuada a sus necesidades clínicas, en las dosis correspondientes, durante un período de tiempo adecuado y al menor costo posible para ellos y la comunidad $^{28}$. Este concepto involucra la eficacia que tienen los fármacos para cambiar la historia natural de la enfermedad para la cual están siendo indicados, y la seguridad que conlleva su uso; aspectos que se pueden evidenciar en su relación beneficio/riesgo.

En Argentina, el programa Remediar, al que se hizo mención en este trabajo, busca incorporar al listado de medicamentos que entrega regularmente en sus botiquines, solo aquellos fármacos que han validado su eficacia y seguridad y tienen el menor costo de mercado ${ }^{29}$. Sin embargo, la cantidad de medicamentos dispensados por este programa solo cubre una parte de los pacientes con cobertura pública exclusiva, y el resto, deben buscar opciones en cada provincia y cada distrito municipal según el lugar geográfico en el que residan. La falta de un listado único recomendado por las autoridades sanitarias nacionales y la interpretación desigual que realizan los distintos responsables en cada dependencia, generan una inequidad en la cobertura y en el acceso a los medicamentos por parte de la población. 
Por otro lado, la Seguridad Social en Argentina, al ser muy heterogénea y estar compuesta por cientos Obras Sociales (aun debiendo cumplir con un PMO), no siguen la misma lógica a la hora de seleccionar los medicamentos que deben cubrir. En muchos casos, como se puede observar en los resultados de este estudio, los fármacos cubiertos no siempre tienen probada eficacia, $o$ no son los más seguros, o bien no son los menos onerosos, por lo tanto no cumplen con los preceptos de URM de la OMS.

Mayoritariamente, para aumentar el acceso, las Obras Sociales y algunos financiadores del sub-sector público de salud, históricamente han decidido como estrategia de gestión, negociar para bajar el precio de una amplia cartilla de medicamentos, sin diferenciar entre aquellos que son poco eficaces o inseguros, de aquellos que no lo son, con lo cual, queda claro que se está priorizando lo económico por sobre lo sanitario y lo racional. Ejemplo de ello se demostró en el año 2018, cuando la gestión de PAMI logró bajar globalmente un $5 \%$ el precio de los medicamentos, hecho que tomó estado público destacándose este logro en las primeras planas de la prensa nacional y regional ${ }^{30,31}$. No obstante, el dato oculto en esta gestión es que se siguió pagando sin discriminar lo racional y lo irracional. Y aún peor, en el caso de un afiliado/jubilado que sea hipertenso, hipotiroideo e hipercolesterolémico debía seguir pagando de bolsillo gran porcentaje de su jubilación en concepto medicamentos que tal vez no incidían positivamente en la evolución de su salud, limitando el acceso a otros bienes esenciales como los alimentos. Esta conducta de los financiadores es riesgosa, sobre todo cuando se trata de patologías crónicas que por no tener síntomas, ponen a los pacientes entre la disyuntiva de alimentarse o pagar por el tratamiento de patologías asintomáticas que no advierten clínicamente tener, aspecto que puede llevar a una reducción en el acceso y en la adherencia los tratamientos; exponiendo a los pacientes a complicaciones severas asociadas a estas enfermedades mal controladas ${ }^{32}$.

La mejor estrategia sería entonces seleccionar mejor aquellos fármacos, por los que se va a pagar, cubriendo el mayor porcentaje posible; $y$ excluir de la cartilla de cobertura aquellos medicamentos que no agregan un beneficio probado para cambiar la historia natural de la enfermedad o bien que tienen un dudoso perfil de seguridad.

Es oportuno mencionar que en la falta de acceso, no solamente median los problemas eco- nómicos, sino deben considerarse como causales otras barreras ${ }^{33}$. Aun con un programa de distribución gratuita de los medicamentos en atención primaria como fue el Programa Remediar en Argentina, se observa que la continuidad en los tratamientos anuales de las enfermedades crónicas es sumamente infrecuente ${ }^{34}$. Solo un bajo porcentaje de las personas hipertensas o diabéticas tratadas con fármacos de distribución gratuita, retiran su medicación regularmente y con continuidad durante todos los doce meses del año. Existen entonces barreras que deben tenerse en cuenta como las culturales, geográficas, de oportunidades, que hacen que los pacientes, aun teniendo disponible en su Centro de Salud la medicación gratuita, no la retiran y pierden la continuidad y la adherencia terapéutica ${ }^{34}$. Algunas de estas barreras tienen relación con los propios individuos, sus hogares y la comunidad; con la prestación de servicios de salud; con el nivel del sector salud; con las políticas públicas transversales a los sectores; o con aspectos relacionados al nivel regiona ${ }^{35}$.

Todo indica que resulta necesario considerar otros modelos de cobertura, dispensa y monitoreo que garanticen un mayor acceso y adherencia a terapéutica prescripta ${ }^{36}$.

Con respecto a las estrategias de los financiadores, podemos decir que el mayor problema de Argentina, es aún carecer de un listado único de medicamentos esenciales al cual pudieran referenciarse tanto el sub-sector público con la seguridad social. Si bien cada uno de los sectores tiene de manera formal o informal un listado que orienta la cobertura; en la mayoría de los casos, los fármacos que allí figuran no han sido seleccionados en base a las pautas del uso racional, sino en la demanda tanto de los pacientes como de los prescriptores.

De lo expuesto hasta el momento, se desprende que el gran desafío planteado a los diferentes sub-sectores del sistema de salud es saber cuáles son los bienes que se deben cubrir y qué beneficios reales tendrán los afiliados o los ciudadanos al acceder a los mismos.

No debe ser el precio ni la presión de los prescriptores lo que inicie el proceso de selección sino que deben ser las ventajas que traen aparejadas el uso del fármaco en términos de modificación de la historia natural de la enfermedad en los destinatarios. Una mayor racionalidad en la cobertura de medicamentos seguramente incrementará el acceso a estos bienes para todos los argentinos. 
En el presente trabajo, se ha brindado una descripción de la organización del Sistema de Salud Argentino y un análisis de los diferentes Sub-Sectores que lo componen, basándose en determinar cómo los beneficiarios acceden a un bien esencial como es el medicamento.

Del análisis realizado se desprende que existe una disparidad en la forma en la cual los argentinos acceden a los fármacos. Las causas que explican esa situación son múltiples. Una de ellas es la ausencia de un listado único de referencia para el país, que hace que cada sector y cada financiador dentro de los sectores, seleccione arbitrariamente los fármacos por los que pagará.

En el sub-sector público, un elemento que juega un rol primordial en el acceso, es el Federalismo existente en Argentina. Cada provincia tiene la potestad de decidir qué tipo de cobertura debe brindar, aspecto que profundiza la desigualdad en la población a acceder a los medicamentos necesarios para afrontar la prevención o el tratamiento de las patologías prevalentes que la afectan. Un ciudadano argentino con cobertura pública exclusiva, por el simple hecho de haber nacido en una provincia $u$ otra, o en un municipio u otro, no tendrá las mismas oportunidades de acceso a determinados fármacos.

Por su parte, la Seguridad Social en Argentina es muy heterogénea, y por ello existen grandes diferencias entre los beneficiarios del sector, a la hora de acceder a los medicamentos.

Por otro lado, en el Sub-Sector Privado, el aspecto que genera mayores inequidades en el acceso son los distintos planes de cobertura según la capacidad de pago de quien contrate el servicio. Por esta razón, en la medicina prepaga, la cartera de beneficiarios fluctúa siguiendo los vaivenes económicos del país.

Por los elementos expuestos, resulta necesario consensuar entre los distintos Sub-Sectores de salud de la Argentina, estrategias destinadas a adoptar un algoritmo de toma de decisiones comunes, en pos de elegir la mejor opción terapéutica que garantice el acceso de toda la población a estos bienes esenciales que permitan modificar la historia natural de las enfermedades.

\section{Referencias}

1. Bonomi PU. Under the cope of heaven. Religion, society and politics in Colonial America. New York: Oxford University Press; $1986.12 \mathrm{p}$.

2. Sanchez-Castañeda A. Las transformaciones del derecho del trabajo. Mexico, UNAM; 2006; 1:23-28.

3. DCOMM. De Bismarck a Beveridge: seguridade social para todos. 2009; 67:11-34.

4. Belmartino S, Bloch C. El sistema de servicios de salud en Argentina. En: González-Pérez GJ, Vega-López MG, Romero-Valle S, coordinators. Los sistemas de salud en Iberoamérica de cara al siglo XXI. Guadalajara, México: Universidad de Guadalajara, Mexico; 2004. $1-32$.

5. Organización Panamericana de la Salud (OPS). Programa de Organización y Gestión de Sistemas y Servicios de Salud. Perfil del sistema de servicios de salud. República Argentina. Washington, DC: OPS; 2001.

6. Belló M, Becerril-Montekio VM. Sistema de salud de Argentina. Salud Pública de México 2011; 53:s96-s109.
7. Belmartino S, Bloch C. Desregulación/privatización. La relación entre financiación y provisión de servicios en la reforma de la seguridad social médica argentina. Cuadernos Médico Sociales 1998; 73:61-80.

8. Cendali F, Pzo L. Politicas de Salud Pública Argentina: Comparación de dos modelos. V Jornadas de Sociologia de la Universidad Nacional de La Plata. Facultad de Humanidades y Ciencias de la Educacion. 2009. [acceso 2020 Jun 3]. Disponible en: www.aacademica. org/000-096/249.

9. Centrángolo O, Goldschmit A. Obras Sociales en Argentina. Origen y situacion actual de um sistema altamente desigual. CECE 2018; 5-19. [acceso 2020 Jun 3]. Disponible en: http://fcece.org.ar/wp-content/uploads/informes/obras-sociales-argentina.pdf

10. Programa Médico Obligatorio (PMO). Superintendencia de Servicios. Buenos Aires: Ministerio de Salud de la Nacion, Argentina; 2020. [acceso 2020 Jun 3]. Disponible en: https://www.sssalud.gob.ar/novedades/archivos/ documentos/PMO.pdf 
11. Belmartino S. Una década de reforma de la atención médica en Argentina. Salud Colectiva 2005; 21:155171.

12. Alonso G. La política de reforma del sistema de obras sociales argentino: un reiterado modelo de gestión estatal y de acción sindical. Estudios Sociológicos 1996; XIV(42):745-774.

13. PROAPS-REMEDIAR. Análisis del Programa. Bol Proaps-Remediar 2004; (13):5-9.

14. Maceira D. Análisis del Programa Remediar. Notas sobre Evaluación y Seguimiento. Centro de Estudios de Estado y Sociedad (CEDES). 2005; 2:13-21. [acceso 2020 Jun 3]. Disponible en: https://www.danielmaceira.com.ar/wp-content/uploads/2014/07/Maceira -Apella-Barbieri-EvalRemediar-final-Julio2005.pdf

15. Belmartino $S$. Los valores vinculados a equidad en la reforma de la atención médica en Argentina. Cad Saude Publica 2002; 18(4):1067-1076.

16. MSAL. 4ta Encuesta Nacional de Factores de Riesgo (ENFR). Ministerio de Salud de la Nacion, Argentina, 2019. [acceso 2020 Jun 3]. Disponible en: https:// bancos.salud.gob.ar/sites/default/files/2020-01/4ta -encuesta-nacional-factores-riesgo_2019_principales -resultados.pdf

17. Lawrynowicz A, Alonso V. El tratamiento de la diabetes mellitus en la normativa sanitaria argentina: una sistematización. Rev Argent Salud Publica 2013; 4(17):39-44.

18. Orchuela J. Acceso y Programas de suministro de medicamentos y vacunas del Minsiterio de Salud de la provincia de Buenos Aires desde los años 90. V Jornadas de Sociología de la UNLP. Universidad Nacional de La Plata. Facultad de Humanidades y Ciencias de la Educación. Departamento de Sociología, La Plata. 2008. [acceso 2020 Jun 3]. Disponible en: https://www.aacademica.org/000-096/255

19. Elorza ME, Moscoso NS, Ripari N. Evaluación de políticas públicas de provisión de fármacos para diabetes mellitus tipo 2 en Argentina: estudio de caso. Salud Colectiva 2021; 8(1):35-45.

20. Marin GH, Cañas M, Homar C et al. Usage of Drug Prescriptions through the REMEDIAR Program in the Province of Buenos Aires, Argentina. Lat Am J Pharm 2008; 27(4):535-542.

21. Brusa AF, Armando PD, Naeko Uema S. Diabetic patients without health coverage: drug use, adherence and complications arising from their underlying disease. Rev Salud Publica 2013; XVII(2):53-62.

22. Brusa AF. Pacientes diabéticos sin cobertura de salud: utilización de medicamentos, adherencia y complicaciones derivadas de su patología de base. Repositorio Digital UNC. [acceso 2020 Jun 3]. Disponible en: https://rdu.unc.edu.ar/

23. Marro MJ; Cardoso AM, Leite I. . Desigualdades regionales en la mortalidad por diabetes mellitus y en el acceso a la salud en Argentina. Cad Saude Publica 2017; 33(9):e00113016.

24. Migueles L, Dozo I, Hasdeu S, Alvarez J, Gifre J, Closa C, Agusto S. Accesibilidad a medicamentos oncológicos. Análisis comparativo según jurisdicción de residencia de la población de cobertura estatal, abordaje cuantitativo y cualitativo. Argentina; 2006-2012. MSAL; Boletin 12. [acceso 2020 Jun 3]. Disponible en: http://200.80.230.14/bitstream/handle/00261/689/Bolet\%C3\%ADn\%2012\%20-\%20Accesibilidad_a_medicamentos_oncologicos.pdf?sequence $=1$ \&isAllowed $=$
25. PAMI. Listado de precios de medicamentos para afiliados. 2021. [acceso 2020 Jun 3]. Disponible en: https:// datos.pami.org.ar/dataset/medicamentos-para-afiliados

26. Dorati C, Mordujovich P, Marin GH., Buschiazzo H, Rojas Cortez R, Alfonso MJ. PAHO Journal 2021, en prensa.

27. Marin GH, Marin L, Agüero D, Pagnotta M, Marin G, Blanco L, Budgetary Impact of Covering "Me-Too" Drugs from Social Security: Buenos Aires State Case Report. J Drug Deliv Ther 2020; 10(3-s):74-80.

28. Manual Farmacêutico Argentino Kairos. 2021. [acceso 2020 Jun 3]. Disponible en: https://ar.kairosweb.com/

29. World Health Organization (WHO). Conference of Experts on the Rational Use of Drugs (1985: Nairobi). The rational use of drugs: report of the Conference of Experts, Nairobi, 25-29 November 1985. Geneva: WHO; 1987. [acceso 2020 Jun 3]. Disponible en: https://apps.who.int/iris/handle/10665/37174

30. Ministerio de Salud (MSAL). Guía de Medicamentos Esenciales en el Primer Nivel de Atención. Programa REMEDIAR 2020. [acceso 2020 Jun 3]. Disponible en: https://www.argentina.gob.ar/salud/remediar/guiamedicamentos-esenciales-pna

31. PAMI. Resumen de Gestión 2018. [acceso 2020 Jun 3]. Disponible en: https://www.pami.org.ar/pdf/memoria-pami-2018.pdf

32. El Cronista. Pami logró acuerdo para bajar precios de medicamentos com multinacionales. 2018. [acceso 2020 Jun 3]. Disponible en: www.cronista.comhttps:// www.cronista.com/negocios/Pami-logro-acuerdo -para-bajar-precios-de-medicamentos-con-multinacionales-20180323-0062.html

33. World Health Organization (WHO). Global status report on non-communicable diseases. Geneva: WHO; 2012.

34. Levesque JF, Harris MF, Russell G. Patient-centred access to health care: conceptualising access at the interface of health systems and populations. Int J Equity Health 2013 11; 12:18.

35. Marin GH, Canas M, Homar C, Perrotta M. Drug utilization from Program REMEDIAR in Buenos Aires, Argentina. Lat Am J Pharm 2008; 27:535-542.

36. Bigdeli M, Jacobs B, Tomson G, Laing R, Ghaffar A, Dujardin B, Van Damme W. Access to medicines from a health system perspective. Health Policy Planning 2013; 28(7):692-704.

37. Marín GH, Rivadulla P, Vázquez A, Juárez D, GIS. Interdisciplinary health project: a model based in home care makes the difference. An An Sist Sanit Navar 2006; 29(1):107-118.

Artículo presentado en $08 / 06 / 2020$

Aprobado en 20/04/2021

Versión final presentada en 22/04/2021

Editores jefes: Romeu Gomes, Antônio Augusto Moura da Silva 